\title{
Anti-magic labeling for Boolean graph of path BG(P_n), $(n \geqslant 4)$
}

\author{
T. Subhramaniyan ${ }^{1^{*}}$ and S. Suruthi ${ }^{2}$ \\ ${ }^{1}$ Department of Mathematics, Guru Nanak College, Chennai, India \\ ${ }^{2}$ Department of Mathematics, Dhanraj Baid Jain College, Chennai, India \\ *Corresponding Author: thangasubhramaniyan@gmail.com, Tel.: 9444896085
}

Available online at: www.isroset.org

Accepted 12/Aug/2018, Online 30/Aug/2018

\begin{abstract}
A graph G is anti-magic if there is a labelling of $\mathrm{G}$ is a one-to-one mapping taking the edges onto $1,2, \ldots . .,|\mathrm{E}|$ such that the sum of the labels assigned to edges incident to distinct vertices are different. A conjecture of Hartsfield and Ringel states that every connected graph different from K_2 is anti-magic. Our main result validates this conjecture for Boolean graph of path $P_{-}(n)(n \geqslant 4)$.
\end{abstract}

Keywords-Boolean graph BG(G) Anti-magic Labeling

\section{INTRODUCTION}

Suppose $G(V, E)$ is a graph and let $E_{G}(v)$ be the set of edges of $G$ incident tov, for each vertex $v$ of $G$. We shall write $E(v)$ for $E_{G}(v)$. Let $f: E \rightarrow\{1,2, \ldots,|E|\}$ be a bijective mapping. The vertex-sum $\varphi_{f}(v)$ at $v$ is defined as $\varphi_{f}(v)=\sum_{\varepsilon \in E(v)} f(e)$. For any two distinct vertices u, $v$ of G, $\varphi_{f}(v) \neq \varphi_{f}(u)$ gives an anti-magic labeling of G. A graph $\mathrm{G}$ is called anti-magic if $\mathrm{G}$ has an anti-magic labeling. The problem of anti-magic labeling of graphs was introduced by Hartsfield and Ringel [4]. They conjectured that all graphs with no single edge component are anti-magic. Graph Labeling has many applications in coding theory, $X$-ray crystallography, radar, astronomy, circuit design, communication network addressing, and data base management.

\section{CONJECTURE 1}

[4] Every connected graph different from $K_{2}$ is anti-magic. This conjecture is still open. Interestingly, the graph $K_{2}$ can be regarded as a tree on two vertices. Thus, if we restrict ourselves to trees, the above conjecture holds. Hartsfield and Ringel proved that paths, cycles and complete graph $K_{\mathrm{n}}$, $(n \geq 3)$ are anti-magic. Recently, Alon et al. [1] have proved that the conjecture is true for some classes of dense graphs. They have shown that all dense graphs with $(n \geq 4)$ vertices and minimum degree $\Omega(\log n)$ are anti-magic. They also proved that if $G$ is a graph with $(n \geq 4)$ vertices and the maximum degree $\Delta(G) \geq 4 n-2$, then $G$ is anti-magic and all complete bipartite graphs except $K_{2}$ are anti-magic. Antimagic labeling of the Cartesian product of graphs was studied in [7]; if $G$ is a regular anti-magic graph then for any graph $\mathrm{H}$, the Cartesian product $\mathrm{H} \times \mathrm{G}$ is anti-magic. It was proved in [4] that 2-regular graphs are anti-magic and proved in [6] that 3-regular graphs are anti-magic. As a consequence, if $\mathrm{G}$ is 2-regular or 3-regular then for any graph $\mathrm{H}, \mathrm{H} \times \mathrm{G}$ is anti-magic. In this paper, we extend anti-magic labeling to Boolean Graph of path.

\section{DEFINITION}

Boolean graph $\mathrm{BG}(\mathrm{G})$ is a graph with vertex set $\mathrm{V}(\mathrm{G}) \cup E(G)$ and two vertices in $B G(G)$ are adjacent if and only if they correspond to two adjacent vertices of $G$ or to a vertex and non - incident edge of $G$.

\section{THEOREM}

The Boolean graph of path $\mathrm{BG}\left(\mathrm{P} \_n\right),(\mathrm{n} \geq 4)$ is anti-magic

Proof: Let $P_{n}$ be the path with vertices $v_{1}, v_{2}, v_{a}, \ldots, v_{n}$. By the definition of Boolean graph $B G\left(P_{n}\right)$ the vertex set is given by

$V\left(B G\left(P_{n}\right)\right)=\left\{v_{i} ; 1 \leq i \leq n\right\} \cup\left\{u_{j} ; 1 \leq j \leq n-1\right\}$ and the edge set is given by

$$
E\left(B G\left(P_{n}\right)\right)=\left\{v_{i} v_{i+1} ; 1 \leq i \leq n-1\right\} \cup\left\{u_{j} u_{j+1} ; 1 \leq j \leq\right.
$$

We discuss Boolean graph of path in two cases.

Case $(\mathbf{a}): \mathrm{n} \equiv 1(\bmod 2)$

Label the vertices of $B G\left(P_{n}\right)$ using the function $f: E \rightarrow N$ as follows:

$\mathrm{f}\left(\mathrm{v}_{\mathrm{i}} \mathrm{v}_{\mathrm{i}+1}\right)=\mathrm{i} ; \mathrm{i}=1,2, \ldots, \mathrm{n}$.

$f\left(u_{j} u_{j+1}\right)=2 n-j ; j=1,2, \ldots, n-1$. 
$\mathrm{f}\left(\mathrm{v}_{\mathrm{i}} \mathrm{u}_{\mathrm{j}}\right)=(\mathrm{n}-1)(\mathrm{i}+1)+\mathrm{j}$ if $\mathrm{i}<\mathrm{j}$, where $1 \leq \mathrm{i} \leq \mathrm{n}+1 \& 2 \leq \mathrm{j}$ $\leq \mathrm{n}$.

$\mathrm{f}\left(\mathrm{v}_{\mathrm{i}} \mathrm{u}_{\mathrm{j}}\right)=(\mathrm{n}-1)(\mathrm{i}-1)+\mathrm{n}+\mathrm{j} \quad$ if $\mathrm{i}>\mathrm{j}$, where $\mathrm{j}=1,2, \ldots, \mathrm{n}$ \& $\mathrm{j}<\mathrm{i} \leq \mathrm{n}+1$

The induced function $f^{*}: V \rightarrow N$ such that

$f^{*}\left(v_{i}\right)=\Sigma_{u_{j} \in \operatorname{end}\left(v_{i}\right)} f\left(v_{i} u_{j}\right)$

We consider the case when labels of vertices are distinct.

Subcase (i): when $i=1$ and $j>i$.

$$
\begin{aligned}
\mathrm{f}^{*}\left(\mathrm{v}_{\mathrm{i}}\right) & =\mathrm{f}\left(\mathrm{v}_{\mathrm{i}} \mathrm{v}_{\mathrm{i}+1}\right)+\sum_{\substack{j=2 \\
i<j}}^{n} f\left(v_{i} u_{j}\right) \\
& =\mathrm{f}\left(\mathrm{v}_{\mathrm{i}} \mathrm{v}_{\mathrm{i}+1}\right)+\sum_{j=2}^{n}[(n-1)(i+1)+j] \\
\mathrm{f}^{*}\left(\mathrm{v}_{1}\right) & =\mathrm{f}\left(\mathrm{v}_{1} \mathrm{v}_{2}\right)+\sum_{j=2}^{n}[(n-1)(1+1)+j] \\
& =1+\sum_{j=2}^{n}[(2 n-2)+j] \\
& =1+(\mathrm{n}-1)(2 \mathrm{n}-2)+\left[\frac{n(n+1)}{2}-1\right] \\
& =1+(\mathrm{n}-1)(2 \mathrm{n}-2)+\frac{n^{2}+n-2}{2} \\
& =\frac{1}{2}\left[2+4 \mathrm{n}^{2}-4 \mathrm{n}-4 \mathrm{n}+4+\mathrm{n}^{2}+\mathrm{n}-2\right] \\
\mathrm{f}^{*}\left(\mathrm{v}_{1}\right) & =\frac{1}{2}\left[5 \mathrm{n}^{2}-7 \mathrm{n}+4\right]
\end{aligned}
$$

Sub case (ii): When $\mathrm{i}=2,3, \ldots, \mathrm{n}$

$$
\begin{aligned}
& \begin{aligned}
\mathrm{f}^{*}\left(\mathrm{v}_{\mathrm{i}}\right) & =\mathrm{f}\left(\mathrm{v}_{\mathrm{i}-1} \mathrm{v}_{\mathrm{i}}\right)+\mathrm{f}\left(\mathrm{v}_{\mathrm{i}} \mathrm{v}_{\mathrm{i}+1}\right)+\sum_{\substack{j=1 \\
j \neq i-1, i}}^{n} f\left(v_{i} u_{j}\right) \\
& =\mathrm{i}+\mathrm{i}-1+\sum_{\substack{j=1 \\
i>j}}^{i-2} f\left(v_{i} u_{j}\right)+\sum_{\substack{j=i+1 \\
i<j}}^{n} f\left(v_{i} u_{j}\right) \\
& =2 \mathrm{i}-1+ \\
\sum_{j=1}^{i-2}[(n-1)(i-1)+n+j]+\sum_{j=i+1}^{n}[(n-1)(i+1)+j] & (i-2)(i-1) \\
& \left.=2 \mathrm{i}-1+(\mathrm{i}-2)[(\mathrm{n}-1)(\mathrm{i}-1)+\mathrm{n}]+\frac{(i-1)}{2}\right]
\end{aligned} \\
& (\mathrm{n}-\mathrm{i})[(\mathrm{n}-1)(\mathrm{i}+1)]+\left[\frac{n(n+1)}{2}-\frac{i(i+1)}{2}\right]
\end{aligned}
$$

$$
=2 \mathrm{i}-1+(\mathrm{i}-2)[\mathrm{ni}-\mathrm{n}-\mathrm{i}+1+\mathrm{n}]+\frac{i^{2}-3 i+2}{2}+
$$

$(\mathrm{n}-\mathrm{i})(\mathrm{ni}+\mathrm{n}-\mathrm{i}-1)+\frac{n^{2}+n-i^{2}-i}{2}$

$$
=2 i-1+n i^{2}-i^{2}+i-2 n i+2 i-2+\frac{i^{2}-3 i+2}{2}+
$$

$$
\begin{gathered}
\mathrm{n}^{2} \mathrm{i}+\mathrm{n}^{2}-\mathrm{ni}-\mathrm{n}-\mathrm{ni}^{2}-\mathrm{ni}+\mathrm{i}^{2}+\mathrm{i}+\frac{n^{2}+n-i^{2}-i}{2} \\
=\frac{1}{2}\left[8 \mathrm{i}-4-8 \mathrm{ni}+2 \mathrm{n}^{2} \mathrm{i}+3 \mathrm{n}^{2}-\mathrm{n}\right] \\
\mathrm{f}^{*}\left(\mathrm{v}_{\mathrm{i}}\right)=\frac{1}{2}\left[\left(2 \mathrm{n}^{2}-8 \mathrm{n}+8\right) \mathrm{i}+\left(3 \mathrm{n}^{2}-\mathrm{n}-4\right)\right]
\end{gathered}
$$

Subcase (iii): When $i=n+1$ and $j>i$

$$
\begin{aligned}
\mathrm{f} *\left(\mathrm{v}_{\mathrm{i}}\right) & =\mathrm{f}\left(\mathrm{v}_{\mathrm{i}-1} \mathrm{v}_{\mathrm{i}}\right)+\sum_{\substack{j=1 \\
i>j}}^{n-1} f\left(v_{i} u_{j}\right) \\
& =\mathrm{f}\left(\mathrm{v}_{\mathrm{i}-1} \mathrm{v}_{\mathrm{i}}\right)+\sum_{j=1}^{n-1}[(n-1)(i-1)+n+j] \\
\mathrm{f}^{*}\left(\mathrm{v}_{\mathrm{n}+1}\right) & =\mathrm{f}\left(\mathrm{v}_{\mathrm{n}} \mathrm{v}_{\mathrm{n}+1}\right)+\sum_{j=1}^{n-1}[(n-1)(n+1-1)+n+j] \\
& =\mathrm{n}+\sum_{j=1}^{n-1}[(n-1) \cdot n+n+j] \\
& =\mathrm{n}+\sum_{j=1}^{n-1}\left[n^{2}-n+n+j\right] \\
& =\mathrm{n}+\sum_{j=1}^{n-1}\left[n^{2}+j\right] \\
\mathrm{f}^{*}\left(\mathrm{v}_{\mathrm{n}+1}\right) & =\frac{1}{2}\left[2 \mathrm{n}^{3}-\mathrm{n}^{2}+\mathrm{n}\right] \\
& =\frac{1}{2}+(\mathrm{n}-1) \cdot \mathrm{n}^{2}+\frac{(n-1) \cdot n}{2} \\
& \\
&
\end{aligned}
$$

Consider the case when labels of edges are distinct.

Subcase (iv): When $j=1$ and $i>j$

$$
\begin{aligned}
\mathrm{f}^{*}\left(\mathrm{u}_{\mathrm{j}}\right) & =\mathrm{f}\left(\mathrm{u}_{\mathrm{j}} \mathrm{u}_{\mathrm{j}+1}\right)+\sum_{\substack{i=j+2 \\
i>j}}^{n+1} f\left(v_{i} u_{j}\right) \\
& =2 \mathrm{n}-\mathrm{j}+\sum_{i=j+2}^{n+1}[(n-1)(i-1)+n+j]
\end{aligned}
$$




$$
\begin{aligned}
& \mathrm{f}^{*}\left(\mathrm{u}_{1}\right)=(2 \mathrm{n}-1)+\sum_{i=3}^{n+1}[(n-1) i-n+1+n+1] \\
& =(2 \mathrm{n}-1)+\sum_{i=3}^{n+1}[(n-1) i+2] \\
& =(2 \mathrm{n}-1)+(\mathrm{n}-1) 2+(\mathrm{n}-1) \\
& {\left[\frac{(n+1)(n+2)}{2}-\frac{2.3}{2}\right]} \\
& =2 \mathrm{n}-1+2 \mathrm{n}-2+\frac{(n-1)\left(n^{2}+3 n+2\right)}{2}-3(n-1) \\
& =\frac{1}{2}\left[8 n-6+n^{3}+3 n^{2}+2 n-n^{2}-3 n-2-6 n+6\right] \\
& \mathrm{f}^{*}\left(\mathrm{u}_{1}\right)=\frac{1}{2}\left[\mathrm{n}^{3}+2 \mathrm{n}^{2}+\mathrm{n}-2\right]
\end{aligned}
$$

Subcase (v): When $j=2,3, \ldots, n-1$

$$
\begin{aligned}
& \mathrm{f}^{*}\left(\mathrm{u}_{\mathrm{j}}\right)=\mathrm{f}\left(\mathrm{u}_{\mathrm{j}-1} \mathrm{u}_{\mathrm{j}}\right)+\mathrm{f}\left(\mathrm{u}_{\mathrm{j}} \mathrm{u}_{\mathrm{j}+1}\right)+\sum_{\substack{i=1 \\
i \neq j, j+1}}^{n+1} f\left(v_{i} u_{j}\right) \\
& =(2 \mathrm{n}-\mathrm{j}+1)+(2 \mathrm{n}-\mathrm{j})+ \\
& \sum_{\substack{i=1 \\
i<j}}^{j-1} f\left(v_{i} u_{j}\right)+\sum_{\substack{i=j+2 \\
i>j}}^{n+1} f\left(v_{i} u_{j}\right) \\
& =4 \mathrm{n}-2 \mathrm{j}+1+ \\
& \sum_{i=1}^{j-1}[(n-1)(i+1)+j]+\sum_{i=j+2}^{n+1}[(n-1)(i-1)+n+j] \\
& =4 \mathrm{n}-2 \mathrm{j}+1+\sum_{i=1}^{j-1}[(n-1) i+(n-1)+j]+\sum_{i=1}^{n+1}[(n-1) i-(n-1)+n+j] \\
& =4 \mathrm{n}-2 \mathrm{j}+1+\frac{(n-1)(j-1) j}{2}+(\mathrm{j}-1)(\mathrm{n}-1+\mathrm{j})
\end{aligned}
$$$$
\left[\frac{(n+1)(n+2)}{2}-\frac{(j+1)(j+2)}{2}\right]+(n-j)(1+\mathrm{j})
$$

$$
f^{*}\left(u_{j}\right)=\frac{1}{2}\left[n^{3}+2 n^{2}+5 n+4-6 j\right]
$$

Sub case (vi): When $j=n$ and $i<j$

$$
\begin{aligned}
\mathrm{f}^{*}\left(\mathrm{u}_{\mathrm{j}}\right) & =\mathrm{f}\left(\mathrm{u}_{\mathrm{j}-1} \mathrm{u}_{\mathrm{j}}\right)+\sum_{\substack{i=1 \\
i<j}}^{j-1} f\left(v_{i} u_{j}\right) \\
& =2 \mathrm{n}-\mathrm{j}+1+\sum_{i=1}^{j-1}[(n-1)(i+1)+j]
\end{aligned}
$$

$$
\begin{aligned}
\mathrm{f}^{*}\left(\mathrm{u}_{\mathrm{n}}\right) & =2 \mathrm{n}-\mathrm{n}+1+\sum_{i=1}^{n-1}[(n-1)(i+1)+n] \\
& =\mathrm{n}+1+\sum_{i=1}^{n-1}[(n-1) i+n-1+n] \\
& =\mathrm{n}+1+\sum_{i=1}^{n-1}[(n-1) i+2 n-1] \\
& =\mathrm{n}+1+\frac{(n-1)(n-1) \cdot n}{2}(\mathrm{n}-1)(2 \mathrm{n}-1) \\
& =\frac{1}{2}\left[2 \mathrm{n}+2+\mathrm{n}^{3}-2 \mathrm{n}^{2}+\mathrm{n}+4 \mathrm{n}^{2}-2 \mathrm{n}-4 \mathrm{n}+2\right] \\
& =\frac{1}{2}\left[\mathrm{n}^{3}+2 \mathrm{n}^{2}-3 \mathrm{n}+4\right]
\end{aligned}
$$

$\therefore \mathrm{BG}\left(\mathrm{P}_{\mathrm{n}}\right)$ is anti-magic.

Case (b): $\mathrm{n} \equiv 0(\bmod 2)$

Label the vertices of $B G\left(P_{n}\right)$ using the function

$\mathrm{f}: \mathrm{E} \rightarrow \mathrm{N}$ as follows:

$\mathrm{f}\left(\mathrm{v}_{\mathrm{i}} \mathrm{v}_{\mathrm{i}+1}\right)=2(\mathrm{n}-\mathrm{i})+1 ; \mathrm{i}=1,2, \ldots, \mathrm{n}$

$f\left(u_{j} u_{j+1}\right)=2(n-j) ; j=1,2, \ldots, n-1$

$\mathrm{f}\left(\mathrm{v}_{\mathrm{i}} \mathrm{u}_{\mathrm{j}}\right)=(\mathrm{n}-1)(\mathrm{i}+1)+\mathrm{j}$ for $\mathrm{i}<\mathrm{j}$

$=(\mathrm{n}-1)(\mathrm{i}-1)+\mathrm{n}+\mathrm{j}$ for $\mathrm{i}>\mathrm{j}$

The induced function $f^{*}: V \rightarrow N$ such that $f^{*}\left(v_{i}\right)=\sum_{u_{j} \in \operatorname{mbd}\left(v_{i j}\right)} f\left(v_{i} u_{j}\right)$

Consider the case when the labels of vertices are distinct.

Subcase (vii): When $i=1$ and $j>i$

$$
\begin{aligned}
& \mathrm{f}^{*}\left(\mathrm{v}_{\mathrm{i}}\right)=\mathrm{f}\left(\mathrm{v}_{\mathrm{i}} \mathrm{v}_{\mathrm{i}+1}\right)+\sum_{\substack{j=2 \\
i<j}}^{n} f\left(v_{i} u_{j}\right) \\
& =2(\mathrm{n}-\mathrm{i})+1+\sum_{j=2}^{n}[(n-1)(i+1)+j] \\
& \mathrm{f}^{*}\left(\mathrm{v}_{1}\right)=2(\mathrm{n}-1)+1+\sum_{j=2}^{n}[(n-1) 2+j] \\
& =2 \mathrm{n}-1+(\mathrm{n}-1)(2 \mathrm{n}-2)+\frac{n(n+1)}{2}-1 \\
& f^{*}\left(v_{1}\right)=\frac{1}{2}\left[5 n^{2}-3 n\right]
\end{aligned}
$$

Subcase (Viii): When $i=2, \ldots, n-1$

$\mathrm{f}^{*}\left(\mathrm{v}_{\mathrm{i}}\right)=\mathrm{f}\left(\mathrm{v}_{\mathrm{i}-1} \mathrm{v}_{\mathrm{i}}\right)+\mathrm{f}\left(\mathrm{v}_{\mathrm{i}} \mathrm{v}_{\mathrm{i}+1}\right)+\sum_{\substack{j=1 \\ j=i-1, i}}^{n} f\left(v_{i} u_{j}\right)$ 


$$
\begin{aligned}
& =2[\mathrm{n}-(\mathrm{i}-1)]+1+2(\mathrm{n}-\mathrm{i})+1+\sum_{\substack{j=1 \\
i>j}}^{i-2} f\left(v_{i} u_{j}\right)+ \\
& \sum_{\substack{j=i+1 \\
i<j}}^{n} f\left(v_{i} u_{j}\right) \\
& =4 n-4 i+4+ \\
& \sum_{j=1}^{i-2}[(n-1)(i-1)+n+j]+\sum_{j=i+1}^{n}[(n-1)(i+1)+j] \\
& =4 n-4 i+4+ \\
& \sum_{j=1}^{i-2}[(n-1) i-n+1+n+j]+\sum_{j=i+1}^{n}[(n-1) i+n-1+j] \\
& =4 \mathrm{n}-4 \mathrm{i}+4+(\mathrm{i}-2)[(\mathrm{n}-1) \mathrm{i}+1]+\frac{(i-2)(i-1)}{2} \\
& +(\mathrm{n}-\mathrm{i})(\mathrm{ni}-\mathrm{i}+\mathrm{n}-1)+\left[\frac{n(n+1)}{2}-\frac{i(i+1)}{2}\right] \\
& =\frac{1}{2}\left[2 n^{2} i-8 n i-4 i+3 n^{2}+7 n+6\right] \\
& f *\left(v_{i}\right)=\frac{1}{2}\left[\left(2 n^{2}-8 n-4\right) i+\left(3 n^{2}+7 n+6\right)\right]
\end{aligned}
$$

Subcase (iX): when $i=n+1$ and $j<i$

$$
\begin{aligned}
& \mathrm{f}^{*}\left(\mathrm{v}_{\mathrm{i}}\right)=\mathrm{f}\left(\mathrm{v}_{\mathrm{i}-1} \mathrm{v}_{\mathrm{i}}\right)+\sum_{\substack{j=1 \\
i>j}}^{n-1} f\left(v_{i} u_{j}\right) \\
& =2[\mathrm{n}-(\mathrm{i}-1)]+1+\sum_{j=1}^{n-1}[(n-1)(i-1)+n+j] \\
& f^{*}\left(\mathrm{v}_{\mathrm{n}+1}\right)=2[\mathrm{n}-(\mathrm{n}+1-1)]+1+ \\
& \sum_{j=1}^{n-1}[(n-1)(n+1-1)+n+j] \\
& =1+\sum_{j=1}^{n-1}[(n-1) n+n+j] \\
& =1+\sum_{j=1}^{n-1}\left[n^{2}+j\right] \\
& =1+(n-1) n^{2}+\frac{(n-1) \cdot n}{2} \\
& f^{*}\left(v_{n+1}\right)=\frac{1}{2}\left[2 n^{3}-n^{2}-n+2\right]
\end{aligned}
$$

We consider the case when the labels of edges are distinct.

Subcase (x): when $j=1$ and $i>j$

$$
\begin{aligned}
\mathrm{f}^{*}\left(\mathrm{u}_{\mathrm{j}}\right) \quad & \mathrm{f}\left(\mathrm{u}_{\mathrm{j}} \mathrm{u}_{\mathrm{j}+1}\right)+\sum_{\substack{i=j+2 \\
1>j}}^{n+1} f\left(v_{i} u_{j}\right) \\
& =2(\mathrm{n}-\mathrm{j})+\sum_{i=j+2}^{n+1}[(n-1)(i-1)+n+j] \\
\mathrm{f}^{*}\left(\mathrm{u}_{1}\right) & =2(\mathrm{n}-1)+\sum_{i=3}^{n+1}[(n-1)(i-1)+n+1] \\
& =2(\mathrm{n}-1)+\sum_{i=3}^{n+1}[(n-1) i-n+1+n+1] \\
& =2(\mathrm{n}-1)+(\mathrm{n}-1)\left[\frac{(n+1)(n+2)}{2}-\frac{2.3}{2}\right]+(\mathrm{n}- \\
1) 2 & =\frac{1}{2}\left[\mathrm{n}^{3}+2 \mathrm{n}^{2}+\mathrm{n}-4\right]
\end{aligned}
$$

Subcase (xi): when $j=2,3, \ldots, n-1$

$$
\mathrm{f}^{*}\left(\mathrm{u}_{\mathrm{j}}\right)=\mathrm{f}\left(\mathrm{u}_{\mathrm{j}-1} \mathrm{u}_{\mathrm{j}}\right)+\mathrm{f}\left(\mathrm{u}_{\mathrm{j}} \mathrm{u}_{\mathrm{j}+1}\right)+\sum_{\substack{i=1 \\ i \neq j, j+1}}^{n+1} f\left(v_{i} u_{j}\right)
$$$$
=2(\mathrm{n}-\mathrm{j})+2+2(\mathrm{n}-\mathrm{j})+
$$$$
\sum_{\substack{i=1 \\ i<j}}^{j-1} f\left(v_{i} u_{j}\right)+\sum_{\substack{i=j+2 \\ i>j}}^{n+1} f\left(v_{i} u_{j}\right)
$$$$
=4 \mathrm{n}-4 \mathrm{j}+2+
$$$$
\sum_{i=1}^{j-1}[(n-1)(i+1)+j]+\sum_{i=j+2}^{n+1}[(n-1)(i-1)+n+j]
$$$$
=4 n-4 j+2+
$$$$
\sum_{i=1}^{j-1}[(n-1) i+n-1+j]+\sum_{i=j+2}^{n+1}[(n-1) i-(n-1)+n+j]
$$$$
=4 \mathrm{n}-4 \mathrm{j}+2+(\mathrm{n}-1) \frac{(j-1) j}{2}+(j-1)(n-1+j)+
$$$$
(n-1)\left[\frac{(n+1)(n+2)}{2}-\frac{(j+1)(j+2)}{2}\right]+(\mathrm{n}-\mathrm{j})(1+\mathrm{j})
$$

$f^{*}\left(u_{j}\right)=\frac{1}{2}\left[n^{3}+2 n^{2}+5 n+6-10 j\right]$

Subcase (xii): when $j=n$ and $i<j$

$\mathrm{f} *\left(\mathrm{u}_{\mathrm{j}}\right)=\mathrm{f}\left(\mathrm{u}_{\mathrm{j}-1} \mathrm{u}_{\mathrm{j}}\right)+\sum_{\substack{i=1 \\ 1<j}}^{j-1} f\left(v_{i} u_{j}\right)$ 


$$
\begin{aligned}
= & 2(\mathrm{n}-\mathrm{j})+2+\sum_{i=1}^{j-1}[(n-1)(i+1)+j] \\
\mathrm{f}^{*}\left(\mathrm{u}_{\mathrm{n}}\right)= & 2(\mathrm{n}-\mathrm{n})+2+\sum_{i=1}^{n-1}[(n-1) i+n-1+n] \\
& =2+(\mathrm{n}-1) \frac{(n-1) n}{2}+(n-1)(2 n-1) \\
\mathrm{f}^{*}\left(\mathrm{u}_{\mathrm{n}}\right) & =\frac{1}{2}\left[\mathrm{n}^{3}+2 \mathrm{n}^{2}-5 \mathrm{n}+6\right]
\end{aligned}
$$

Hence in all the above cases the labeling of all the vertices and the edges of the Boolean graph of path is anti-magic.

$\therefore \mathrm{BG}\left(\mathrm{P}_{\mathrm{n}}\right)$ is anti-magic.

\section{CONCLUSION}

Finally we conclude that the anti-magic labeling to Boolean Graph of path is anti-magic.

\section{REFERENCES}

[1] N. Alon, G. Kaplan, A. Lev, Y. Roditty, R. Yuster, Dense graphs are antimagic, Journal of Graph Theory 47 (2004) 297-309.

[2] W. Brown, Antimagiclabelings and the antimagic strength of graphs, manuscript, 2008.

[3] D.W. Cranston, Regular bipartite graphs are antimagic, Journal of Graph Theory 60 (3) (2009) 173-182.

[4] N. Hartsfield, G. Ringel, Perals in Graph THeory, Academic Press, INC, Boston, 1990, pp. 108-109. Revised version 1994.

[5] G. Kaplan, A. Lev, Y. Roditty, On zero-sum partitions and antimagic trees, Discrete Mathematics 309 (2009) 2010-2014.

[6] Y. Liang, X. Zhu, Antimagic labeling of regular graphs, manuscript, 2012.

[7] Y. Zhang, X. Sun, The antimagicness of the Cartesian product of graphs, Theoretical Computer Science 410 (2009) 727-735.

[8] Subramanian Arumugam, Mirka Miller, OudonePhanalasy and Joe Ryan, Antimagic labeling of generalized pyramid graphs, ActaMathematicaSinica, English Series, 30, 2, (283).

\section{AUTHORS PROFILE}

T. Subhramaniyan, Associate Professor \& Head, Department of Mathematics, Guru Nanak College, Chennai has an in depth knowledge in Mathematics and a well experienced teacher with 30 years of experience. His field of specialization is Algebra and Graph Theory.

S. Suruthi, Assistant Professor, Department of Mathematics, Dhanraj Baid Jain College, Chennai, India has wide knowledge in Mathematics and a teacher with 3 years of experience. Her field of specialization is Graph Theory.
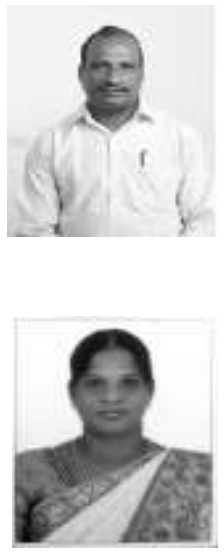